\title{
Neutrino Oscillations in Noisy Media
}

\author{
F. N. Loreti and A. B. Balantekin \\ Department of Physics, University of Wisconsin-Madison, \\ Madison, WI 53706 \\ and \\ Institute for Nuclear Theory, University of Washington, \\ Seattle, WA 98195
}

August 29, 2018

\begin{abstract}
We develop the Redfield equation for delta-correlated gaussian noise and apply it to the case of two neutrino flavor or spin precession in the presence of a noisy matter density or magnetic field, respectively. The criteria under which physical fluctuations can be well approximated by the delta-correlated gaussian noise for the above cases are examined. Current limits on the possible neutrino magnetic moment and solar magnetic field suggest that a reasonably noisy solar magnetic field would not appreciably affect the solar electron neutrino flux. However, if the solar electron density has fluctuations of a few percent of the local density and a small enough correlation length, the MSW effect is suppressed for a range of parameters.
\end{abstract}

\section{INTRODUCTION}

Neutrino oscillations in the presence of matter and magnetic fields have been an area of intense study for approximately the last ten years. In the Mikheyev - Smirnov - Wolfenstein (MSW) effect, electron neutrinos on their journey from the core, are resonantly transformed into muon or tau neutrinos [1, 2]. If neutrinos are Majorana fermions with transition magnetic moments, they can undergo a magnetic resonant transformation into muon or tau antineutrinos [3]. Neutrinos from a supernovae explosion can be transformed from one flavor to another as they pass through the outer part of the star [4]. In many stellar situations, the matter density and/or magnetic fields may fluctuate about a mean value. Some well known examples where fluctuations are likely to exist include the magnetic field and the matter density in the solar convective zone and also the turbulence of the post-supernovae matter which 
has been blown off by the explosion. A general approach to the neutrino oscillations in inhomogeneous matter was developed in Ref. [5]. A Study of matter fluctuations which are not random, but harmonic [6, 7], or occur as a jump-like change in the solar density [8], are available in the literature. Matter currents and density changes effect neutrino flavor oscillations in a similar way and have also been examined in Ref. [7]. Although matter current effects become important only if the velocity is somewhat close to the speed of light, noisy mixing of matter would also mimic a fluctuating matter density. A priori, fluctuations in such fields may be well approximated by random noise added to an average value. In this paper we show how such noise will affect neutrino oscillations for the situation in which the correlation length of the randomly fluctuating part of either the matter density or the magnetic field is small compared with the neutrino oscillation length.

The case of neutrino spin precession in a noisy magnetic field was considered by Nicolaidis for neutrinos in vacuum [9]. The noise was taken to be well approximated by a delta-correlated gaussian distribution with the result that the normal oscillations become damped with a relaxation time of $t_{r e l}=\left(2 \mu^{2}<B_{r}^{2}>\tau_{c}\right)^{-1}$, where $B_{r}$ is the randomly fluctuating part of the magnetic field and $\tau_{c}$ is its correlation time. Enqvist and Semikoz considered neutrino oscillations in randomly fluctuating magnetic field, approximately, by averaging the coefficients in the third order differential equation for the z-component of the neutrino spin, in a constant matter density [10]. Their result suggested that the effect of the randomly fluctuating part of the magnetic field was similar to the effect of a larger (constant) matter density, hence reducing the neutrino precession. Our result is quite different; namely that both a noisy magnetic field and a noisy density (for a constant averaged density) act to depolarize the neutrinos. For a noisy magnetic field, if the probability of transition is greater than one half without the random fluctuations, the inclusion of the random fluctuations will reduce the transition probability, and if the transition probability without the random fluctuations is less than one half, the inclusion of the fluctuations will increase the transition probability. If the randomly fluctuating part of the magnetic field is strong enough or is allowed to act for a long enough time, a complete depolarization of particles-antiparticles will occur. For a noisy density, we find that the MSW transition probability is suppressed. For the case of strongly adiabatic MSW transitions and large fluctuations, the averaged transition probability saturates at one half.

In Section II, we develop the equations governing the time evolution of the averaged probabilities of being found in the Nth level of an N-level system, subject to random and non-random potentials. These equations are derived for the case where the randomly fluctuating part of the field is taken to be a delta-correlated gaussian. In Section III, we show several analytically solvable examples for the case of a two level problem, and numerically examine cases the analytical solutions of which are not instructive. In Section IV, we investigate the conditions under which a real, physical fluctuating field will be well approximated by the equations developed for the delta-correlated gaussian case. We then apply these conditions and examine the 
cases of spin-flavor and flavor precession of neutrinos in the sun. Section V presents a discussion of results and our conclusions.

\section{FORMULATION OF THE APPROACH}

We first consider the general case of an N-level system with time dependent level splitting and transition terms, in the presence of an additional randomly fluctuating term. Since we are interested in the ensemble average of the probability of finding the system in a given level at time t, we consider the density operator defined as,

$$
\hat{\rho} \equiv \psi \otimes \psi^{\dagger}
$$

where,

$$
\psi(t)=\left(\begin{array}{c}
\psi_{1}(t) \\
\psi_{2}(t) \\
\vdots \\
\psi_{N}(t)
\end{array}\right)
$$

Assuming that $\psi$ obeys the Schrödinger-like equation,

$$
i \frac{d}{d t} \psi=\hat{H} \psi
$$

$\hat{\rho}$ obeys the equation,

$$
i \frac{d}{d t} \hat{\rho}=[\hat{H}, \hat{\rho}]
$$

where the Hamiltonian is taken to be linear in fluctuating field, i.e.

$$
\hat{H}=\hat{H}_{0}(t)+B(t) \hat{M}^{\prime}
$$

where $\hat{M}^{\prime}$ is independent of time. Eq. (4) becomes,

$$
i \frac{d}{d t} \hat{\rho}_{I}=\left[\hat{H}_{I}, \hat{\rho}_{I}\right]
$$

where,

$$
\begin{aligned}
\hat{\rho}_{I} & =\hat{U}_{0}^{\dagger} \hat{\rho} \hat{U}_{0}, \\
\hat{M} & =\hat{U}_{0}^{\dagger} \hat{M}^{\prime} \hat{U}_{0}, \\
\hat{H}_{I} & =B(t) \hat{M}
\end{aligned}
$$

and $\hat{U}_{0}$ satisfies,

$$
i \frac{d}{d t} \hat{U}_{0}=\hat{H}_{0} \hat{U}_{0}
$$


This equation can be solved by iteration as,

$$
\begin{aligned}
\hat{\rho}(t) & =\hat{\rho}_{0}-i \int_{0}^{t} d t_{1} B\left(t_{1}\right)\left[\hat{M}\left(t_{1}\right), \hat{\rho}_{0}\right] \\
& -\int_{0}^{t} \int_{0}^{t_{1}} d t_{1} d t_{2} B\left(t_{1}\right) B\left(t_{2}\right)\left[\hat{M}\left(t_{1}\right),\left[\hat{M}\left(t_{2}\right), \hat{\rho}_{0}\right]\right]+\cdots
\end{aligned}
$$

We now assume that $\mathrm{B}(\mathrm{t})$ is such that,

$$
<B(t)>=0, \quad<B\left(t_{1}\right) B\left(t_{2}\right)>=\alpha^{2} f\left(\left|t_{2}-t_{1}\right|\right),
$$

with the average of all higher odd products of B vanishing, and all higher even products given by the sum of all possible products of f's for example,

$$
<B\left(t_{1}\right) B\left(t_{2}\right) B\left(t_{3}\right) B\left(t_{4}\right)>=\alpha^{4}\left[f_{12} f_{34}+f_{13} f_{24}+f_{14} f_{32}\right]
$$

where, $f_{12} \equiv f\left(\left|t_{2}-t_{1}\right|\right)$. This will result in only the even products contributing to $<\hat{\rho}(t)>$.

Further analytic progress can be made if the random potential is a delta correlated gaussian distribution, where $f\left(\left|t_{1}-t_{2}\right|\right)$ becomes $2 \tau \delta\left(t_{1}-t_{2}\right)$. Averaged values of functions of the random field $\mathrm{B}(\mathrm{t})$ can then be expressed as a path integral,

$$
<g(B(t))>=\int_{-\infty}^{\infty} \mathcal{D}[B(t)] g(B) e^{-\int_{0}^{t} d t B^{2} / 2 k}
$$

where,

$$
\mathcal{D}[B(t)]=\prod_{i} d B\left(t_{i}\right) \sqrt{\frac{\Delta t}{2 k \pi}},
$$

and $k=2 \alpha^{2} \tau$. This reproduces the form of the averages of the even power products, and the integrals in Eq. (9) can be explicitly calculated with the result,

$$
\begin{aligned}
<\hat{\rho}_{I}(T)>=\hat{\rho}_{0} & -\alpha^{2} \tau \int_{0}^{T} d t_{1}\left[\hat{M}\left(t_{1}\right),\left[\hat{M}\left(t_{1}\right), \hat{\rho}_{0}\right]\right] \\
& +\alpha^{4} \tau^{2} \int_{0}^{T} d t_{1}\left[\hat{M}\left(t_{1}\right),\left[\hat{M}\left(t_{1}\right), \int_{0}^{t_{1}} d t_{2}\left[\hat{M}\left(t_{2}\right),\left[\hat{M}\left(t_{2}\right), \hat{\rho}_{0}\right]\right]\right]\right]
\end{aligned}
$$

This, however, is just the iterative expansion of the differential equation,

$$
\frac{d}{d t}<\hat{\rho}_{I}(t)>=-\alpha^{2} \tau\left[\hat{M}(t),\left[\hat{M}(t),<\hat{\rho}_{I}(t)>\right]\right] .
$$

One may equally well express this result as,

$$
\frac{d}{d t}<\hat{\rho}(t)>=-\alpha^{2} \tau\left[\hat{M}^{\prime},\left[\hat{M}^{\prime},<\hat{\rho}(t)>\right]\right]-i\left[\hat{H}_{0}(t),<\hat{\rho}(t)>\right] .
$$

Eq. (16), in general, defines a set of $\mathrm{N}^{2}$ coupled first order linear differential equations with the constraint that $\operatorname{Tr} \hat{\rho}=1$. This is a matrix form of the Redfield equation [11]. In the case of neutrinos moving in a varying background, Eq. (16) is equivalent to Eq. (49) in Ref. [5]. 


\section{Analytic Examples}

Perhaps the simplest examples are those for which one could obtain an integral expression for the exact solution for the probabilities of the system being found in the $N^{\text {th }}$ level at time t, and then perform the averaging using Eq. (12). Such an expression is possible, in general, only in the case where all $\mathrm{N}$ levels are degenerate. For example, consider the case in which the Hamiltonian is an $N \times N$ matrix which is of the form $\hat{H}(t)=\left(B_{0}(t)+B_{r}(t)\right) \times \hat{M}$, where $\hat{M}$ has zeros on the diagonal and ones everywhere else. One could, for this case, obtain an integral expression for

the probabilities and then average them. Instead, we take $\hat{H}_{0}(t)=B_{0}(t) \hat{M}$ giving $\hat{U}_{0}(t)=\exp \left(\int_{0}^{t} d t B_{0}(t) \hat{M}\right)$ and use Eq. (15) which becomes,

$$
\frac{d}{d t}<\hat{\rho}_{I}(t)>=-\frac{k(t)}{2}\left[\hat{M},\left[\hat{M},<\hat{\rho}_{I}(t)>\right]\right]
$$

where

$$
k(t) / 2=\alpha^{2}(t) \tau .
$$

Taking the commutator of $\hat{M}$ with $\frac{d}{d t}<\hat{\rho}_{I}(t)>$, and noting that $\hat{M}^{2}=(N-1)+$ $(N-2) \hat{M}$, one obtains,

$$
\frac{d}{d t}\left[\hat{M},<\hat{\rho}_{I}(t)>\right]=-\frac{N^{2}}{2} k(t)\left[\hat{M},<\hat{\rho}_{I}(t)>\right]
$$

This equation is easily solved with the solution,

$$
<\hat{\rho}(T)>=\hat{X}(T)-N^{-2}\left(1-e^{-\frac{N^{2}}{2} \int_{0}^{T} d t k(t)}\right)[\hat{M},[\hat{M}, \hat{X}(T)]]
$$

where $\hat{X}(t)=\hat{U}_{0}(t) \hat{\rho}(0) \hat{U}_{0}^{\dagger}(t)$. This reproduces the result of Nicolaidis for the case where $\hat{M}^{\prime}$ is the Pauli matrix $\sigma_{x}$, and the initial state is $\psi_{1}(0)=1, \psi_{2}(0)=0$.

A second analytically solvable example is a two level system, with an arbitrary time dependent level splitting, in a purely random field. This would correspond to the case of matter-enhanced neutrino spin precession in a noisy magnetic field. In this case, there exists no analytic expression for the probabilities which one could in principle average. We begin by using Eq. (16) with $\hat{H}_{0}=A(t) \sigma_{z}+B_{0}(t) \sigma_{x}$ and $\hat{M}^{\prime}=\sigma_{x}$. Defining $r(t) \equiv \frac{1}{2}\left(<\hat{\rho}_{11}>-<\hat{\rho}_{22}>\right), x=2 \operatorname{Re}\left(<\hat{\rho}_{12}>\right)$ and $y \equiv 2 \operatorname{Im}\left(<\hat{\rho}_{12}>\right)$, one obtains,

$$
\frac{d}{d t}\left(\begin{array}{l}
r \\
x \\
y
\end{array}\right)=-2\left(\begin{array}{ccc}
k & 0 & B_{0} \\
0 & 0 & A \\
-B_{0} & -A & k
\end{array}\right)\left(\begin{array}{l}
r \\
x \\
y
\end{array}\right) .
$$

If $B_{0}=0 \mathrm{Eq} .(21)$ is trivially solved giving,

$$
r(T)=r(0) e^{-2 \int_{0}^{T} d t k(t)},
$$


resulting in,

$$
<\left|\psi_{1}(T)\right|^{2}>=\frac{1}{2}\left(1-e^{-2 \int_{0}^{T} d t k(t)}\right)+\left|\psi_{1}(0)\right|^{2} e^{-2 \int_{0}^{T} d t k(t)} .
$$

This result is surprising in that it is independent of the level splitting and identical to the result for zero level splitting. If $B_{0} \neq 0$, one could still obtain an analytic expression for the averaged probabilities if the level splitting is constant, although such an expression is not instructive due to the complicated nature of third roots. We instead calculate numerically cases of constant level splitting. Figure (1a) through (1d) show the probability $\left|\psi_{1}(t)\right|^{2}$ for $A=0, A=0.5, A=2$ and $A=10$ respectively, for $B_{0}=1$ and $k=0.05$. One observes from these figures that as $A$ is increased, the oscillations become heavily damped while the exponentially decreasing upper envelope remains essentially unchanged. This qualitatively indicates that even when one is far from resonance ( $A=0$ for the case in which $\mathrm{A}$ is time dependent), one can still obtain complete depolarization for such a randomly fluctuating field.

Another possibility occurs if one has the case of a noisy diagonal term with a nonnoisy off-diagonal term, namely $H=\left(A_{0}(t)+A_{r}(t)\right) \sigma_{z}+B_{0}(t) \sigma_{x}$. This case would correspond to a noisy matter density in the context of matter-enhanced neutrino oscillations. Eq. (16) becomes,

$$
\frac{d}{d t}\left(\begin{array}{l}
r \\
x \\
y
\end{array}\right)=-2\left(\begin{array}{ccc}
0 & 0 & B_{0}(t) \\
0 & k & -A_{0}(t) \\
-B_{0}(t) & A_{0}(t) & k
\end{array}\right)\left(\begin{array}{l}
r \\
x \\
y
\end{array}\right) .
$$

If $A_{0}(t)=0$ and $B_{0}$ is time independent, one can obtain an exact solution which, for the initial condition $\psi_{1}(0)=1, \psi_{2}(0)=0$ and $4 B_{0}^{2}>k^{2}$ is,

$$
\left|\psi_{1}(t)\right|^{2}=\frac{1}{2}+\frac{1}{2} e^{-k t}\left[\frac{k}{\omega} \sin \omega t+\cos \omega t\right],
$$

where $\omega=\sqrt{4 B_{0}^{2}-k^{2}}$. We again numerically calculate examples in which the nonrandom diagonal term is non-zero. Figures (2a) through $(2 \mathrm{~d})$ show $\left|\psi_{1}(t)\right|^{2}$ for $A_{0}=$ $0, A_{0}=0.5, A_{0}=2$ and $A_{0}=10$ respectively for $B_{0}=1$ and $k=0.05$. In in contrast to the case of a noisy off-diagonal term, in this case one observes that as the oscillations are damped, the exponential relaxation time increases. Thus, if one is far from resonance, the depolarization is very much suppressed, as is physically reasonable.

\section{NEUTRINOS IN NOISY SOLAR FIELDS}

In Appendix I, we detail the expansion of the density matrix in the case where the randomly fluctuating field has a finite correlation time, with the result that Eq. (16) can accurately describe a real physical situation if,

$$
\left(d / d t(\log \alpha(t))+\left[\hat{H}_{0}(t), \hat{M}^{\prime}\right]\right) \tau_{c} \ll 1,
$$


for all $\mathrm{t}$ of interest, where $\tau_{c}$ is the correlation time of the randomly fluctuating field and $\alpha$ is it's root mean square value, which may be time dependent. If this is satisfied for a given problem, $k(t)$ is given by,

$$
k(t)=\beta \alpha^{2} \tau_{c}(t),
$$

where $\beta$ is a factor of order unity, equal to 2 for our choice in Appendix I of a theta function correlation.

\subsection{Spin Precession in a noisy solar magnetic field}

We first consider the case of matter-enhanced spin-flavor precession of Majorana neutrinos with a negligibly small MSW mixing angle. In this case the evolution equation is two by two, and is given by [3],

$$
i \frac{d}{d r}\left(\begin{array}{c}
\psi_{\nu_{e}} \\
\psi_{\bar{\nu}_{\mu}}
\end{array}\right)=\left(\begin{array}{cc}
-\frac{\Delta m^{2}}{4 E}+a_{e} & \mu\left(B(r)+B_{r}(r)\right) \\
\mu\left(B(r)+B_{r}(t)\right) & \frac{\Delta m^{2}}{4 E}-a_{\mu}
\end{array}\right)\left(\begin{array}{c}
\psi_{\nu_{e}} \\
\psi_{\bar{\nu}_{\mu}}
\end{array}\right),
$$

where $\Delta m^{2} \equiv m_{2}^{2}-m_{1}^{2}$ with $m_{i}$ the masses of the mass eigenstate neutrinos, $\mathrm{E}$ is the neutrino energy, $\mu$ is the neutrino transition magnetic moment, $B$ and $B_{r}$ are the magnetic field and its noise, and $a_{e}$ and $a_{\mu}$ are the matter potentials given by,

$$
a_{e}=\frac{1}{\sqrt{2}} G_{F}\left(2 N_{e}-N_{n}\right), \quad a_{\mu}=\frac{-1}{\sqrt{2}} G_{F} N_{n}
$$

where $N_{e}(r)$ and $N_{n}(\mathrm{r})$ are the electron and neutron number densities.

From the discussion in the previous section of a noisy off-diagonal term, we can estimate whether a reasonably noisy magnetic field can effect the transition probability.

In order for the random fluctuations to have much effect, $2 k \Delta r \sim 1$. The condition for this real process to be approximated by a delta-correlated gaussian, namely Eq. (26), results, for a constant rms value of the the magnetic field, in the condition,

$$
\tau_{c} \times\left[-\frac{\Delta m^{2}}{2 E}+\sqrt{2} G_{F}\left(N_{e}-N_{n}\right)\right] \ll 1
$$

Perhaps the most likely place a noisy magnetic field would exist is in the solar convective zone which extends from approximately $0.7 R_{\odot}$ to the surface [12]. The magnitude of the solar magnetic field observed at the surface of the sun can reach local values of several kilogauss in magnetic storms, and it is thought that it may reach values of $100 \mathrm{kG}$ near the bottom of the convective zone [13]. While a large ( $\sim 100 \mathrm{kG})$ field which extends through out a large fraction of the convective zone is not ruled out, such a large, extensive field is thought to be unlikely [14. Field strengths as large as several times $100 \mathrm{kG}$ could exist if limited to extensions of about $10-100 \mathrm{~km}$. In order to estimate the maximum effect of magnetic field fluctuations, 
we will assume that the solar magnetic field from $0.7 R_{\odot}$ to $0.85 R_{\odot}$ can be considered to be randomly fluctuating with zero mean and a rms value of $100 \mathrm{kG}$. We take the neutrino transition magnetic moment to be $\mu=3 \times 10^{-12} \mu_{B}$, in accord with the maximum bound from plasmon decay in pre-helium flash red giant stars [15]. By choosing a value of $\Delta m^{2} / 2 E$, one can determine the maximum $\tau_{c}$ (assumed to be constant) such that Eq. (30) is satisfied throughout the above region in the solar convective zone. Therefore, we take $\tau_{c}$ to given by,

$$
\tau_{c}=0.1 \times\left(\left|-\frac{\Delta m^{2}}{2 E}+\sqrt{2} G_{F}\left(N_{e}-N_{n}\right)\right|_{\max }\right)^{-1} .
$$

Given that Eq. (30) is obeyed, the average electron neutrino probability after traversing this region is given by Eq. (23), with $\psi_{e}(0)=1$, and for small $2 k \Delta r$ the muon antineutrino average probability is approximately given by $k \Delta r$. In Figure (3) we plot the value of $k \Delta r=4<(\mu B)^{2}>\tau_{c}\left(0.15 R_{\odot}\right)$ as a function of $\Delta m^{2} / E$. One observes that the maximum occurs at about $1 \times 10^{-8} \mathrm{eV}^{2} / \mathrm{MeV}$ corresponding to the largest permissible value of $\tau_{c}$. The peak is due to the fact that the permissible values of $\tau_{c}$ are maximized when the neutrino is in resonance somewhere between $0.7 R_{\odot}$ and $0.85 R_{\odot}$. If one wished to satisfy the condition on $\tau_{c}$ for $\Delta m^{2} / E$ less than or equal to $4 \times 10^{-8} \mathrm{eV}^{2} / \mathrm{MeV}$, one would obtain about $3 \%$ muon antineutrinos on average. This is the same order of magnitude as that is produced by resonant transitions in this region of the sun, for comparable magnetic moments and magnetic fields [13, 16, 14].

We would also like to point out that in the case of non-zero mixing between neutrino species in a purely random magnetic field like that considered above, one can derive the exact (assuming Eq. (26) holds) time dependence for the sum of the neutrino probabilities i.e. $\left|\psi_{e}(r)\right|^{2}+\left|\psi_{\mu}(r)\right|^{2}$. The Hamiltonian is given by [3],

$$
\hat{H}=\left(\begin{array}{cc}
H_{L} & 0 \\
0 & H_{R}
\end{array}\right)+\mu B_{r}\left(\begin{array}{cc}
0 & \hat{M}^{\prime} \\
-\hat{M}^{\prime} & 0
\end{array}\right),
$$

where, $\hat{M}^{\prime}=i \hat{\sigma}_{y}$ and,

$$
H_{L, R}=\left(\begin{array}{cc}
-\frac{\Delta m^{2}}{4 E} \cos 2 \theta \pm a_{e} & \frac{\Delta m^{2}}{4 E} \sin 2 \theta \\
\frac{\Delta m^{2}}{4 E} \sin 2 \theta & \frac{\Delta m^{2}}{4 E} \cos 2 \theta \pm a_{\mu}
\end{array}\right)
$$

If one evaluates Eq. (16) one can derive the equation for the sum of the neutrino probabilities. The solution is given by Eq. (23), with $<\left|\psi_{1}(T)\right|^{2}>$ replaced by the sum of the electron and muon neutrino probabilities. Therefore, the same estimates as in Fig. (3) apply to this case but for the sum of the antineutrino averaged probabilities. One should note that the flavor precession will, in general, be changed by the inclusion of the magnetic field. 


\subsection{Flavor Precession with a Noisy Matter Density}

The equation governing matter-enhanced flavor oscillations (for two flavors) is given by,

$$
i \frac{d}{d r}\left(\begin{array}{c}
\psi_{\nu_{e}} \\
\psi_{\nu_{\mu}}
\end{array}\right)=\left(\begin{array}{cc}
-\frac{\Delta m^{2}}{4 E} \cos 2 \theta+\frac{1}{\sqrt{2}} G_{F} N_{e} & \frac{\Delta m^{2}}{4 E} \sin 2 \theta \\
\frac{\Delta m^{2}}{4 E} \sin 2 \theta & \frac{\Delta m^{2}}{4 E} \cos 2 \theta-\frac{1}{\sqrt{2}} G_{F} N_{e}
\end{array}\right)\left(\begin{array}{c}
\psi_{\nu_{e}} \\
\psi_{\nu_{\mu}}
\end{array}\right)
$$

where $\theta$ is the vacuum mixing angle. We investigate the case in which $N e=(1+$ $\beta) \overline{N_{e}}(r)$ where $\beta$ is a random quantity whose rms value is the rms value of the density fluctuations relative to the average density and $\overline{N_{e}}$ is the electron density in the standard solar model. We satisfy Eq. (26) by choosing,

$$
\tau_{c}=0.1 \times\left(\frac{\Delta m^{2}}{2 E} \sin 2 \theta\right)^{-1},
$$

where the logarithmic derivative in Eq. (26) is much smaller for the sun than the term we have kept. We numerically solve Eq. (24) for the electron neutrino survival probability (averaged for infinite distance beyond the surface of the sun), as a function of $\Delta m^{2} / E$ for fixed values of $\sin ^{2} 2 \theta$ and $\sqrt{<\beta^{2}>}$, with $\tau_{c}$ given by Eq. (35). We have used the solar electron density of Bahcall and collaborators. Figures (4a) through (4d) show the electron neutrino survival probability as a function of $\Delta m^{2} / E$ for $\sin ^{2} 2 \theta=0.8,0.1,0.01$ and 0.001 respectively, with $\sqrt{\left\langle\beta^{2}\right\rangle}=0.02$ In these figures, the curve which reaches the smallest values in the center of the plot (from about $\Delta m^{2} / E=1 \times 10^{-6}$ to $\left.1 \times 10^{-5} \mathrm{eV}^{2} / \mathrm{MeV}\right)$ is the probability in the absence of the density fluctuations. One observes a suppression of the flavor transition in this region by as much as $20 \%$ in Fig. (4c). The region of the largest effect (around $\Delta m^{2} / E=$ $1 \times 10^{-5}$ is in agreement with the maximum value of $2 k \Delta r$, where $\Delta r$ is the width of the resonance region. In order to show the sensitivity of the suppression to the size of the fluctuations, we show the same plots as Fig. (4a)-(4d) in Fig. (5a)-(5d), with the exception that the rms fluctuation in the density is doubled to $4 \%$ of the average density. One observes a very large effect in the previously mentioned region, which saturates at a probability of one half, for values near $\Delta m^{2} / E=1 \times 10^{-5} \mathrm{eV}^{2} / \mathrm{MeV}$. One should note that in Figures (4a)-(5d), $\tau_{c}$ was chosen for each value of $\Delta m^{2} / E$ to obey Eq. (35). Thus, the $\tau_{c}$ chosen for $\Delta m^{2} / E=1 \times 10^{-5} \mathrm{eV}^{2} / \mathrm{MeV}$, while permissible for smaller values of $\Delta m^{2} / E$, would not have given as large an effect, and would, for larger values of $\Delta m^{2} / E$ violate Eq. (35).

In Figs. (4) and (5), there appears to be an enhancement of the transition probability for values of $\Delta m^{2} / E>1.5 \times 10^{-5}$. Neutrinos of with these parameter values do not go through a resonant transition in the sun, since a resonant transition would require a density larger than the maximum solar density. However, to a varying extent they are within the second half of their resonant region near the center of the sun. The solar density profile near the center is rather flat and therefore one obtains an enhancement of the transition probability similar to that in Fig. (3c) and (3d). 
One needs to question whether such fluctuations can arise in the sun, and whether the correlation lengths, which can be quite small $\left(\tau_{c}\right.$ for $\Delta m^{2} / E=1 \times 10^{-5} \mathrm{eV}^{2} / \mathrm{MeV}$ and $\sin ^{2} 2 \theta=0.01$ is about $10 \mathrm{~km}$ ) could be realistic. If one were to do a numerical study utilizing a good random number generator, one would not be limited by Eq. (35) and could consider any correlation length. Our purpose is only to show that such effects could be important.

\section{Conclusions}

We have derived a Redfield differential equation for the time dependence of averaged values of functions of the probability of finding an $\mathrm{N}$ level system in the $\mathrm{N}^{\text {th }}$ level after being subjected to a randomly fluctuating field, of the delta-correlated gaussian type. This formalism applies as an approximation to the case where the fluctuating field can be described by a finite correlation time. This approximation is valid if the product of the correlation time and the energy scale of the Hamiltonian is small. Upon applying this to neutrino flavor or spin-flavor precession, we have shown that the probability will relax eventually to a value of one half, if the neutrino spends a sufficiently long time in a medium with randomly fluctuating matter density or a randomly fluctuating magnetic field. In the case of a fluctuating magnetic field, the relaxation is independent of the matter density, assuming the correlation time of the field fluctuations is small compared to the neutrino oscillation length in matter. In the case of fluctuations added to a constant matter density, the probability again will eventually relax to a value of one half, but the relaxation time is greatly increased if one is far from the resonant condition.

We have also examined the case of neutrino spin-flavor precession in the sun, for a purely random magnetic field and no flavor mixing. It appears that the current limits on the neutrino magnetic moment and the guesses concerning the maximum values of the solar magnetic field, combine to give only a small effect on the average electron neutrino flux. When there is flavor mixing and a purely random field, the combined average probabilities of the neutrinos is again a simple exponentially decreasing function and therefore the results of a purely random field and no flavor mixing apply to this case as well. For the case of a randomly fluctuating electron density, the MSW effect can be strongly suppressed for rms fluctuations of $4 \%$ of the local electron density. However, this requires correlation lengths of about $40 \mathrm{~km}$, and seems to give a significant effect only for neutrinos which have their MSW resonant transition deep in the sun.

In spite of these problems, we believe that a numerical study, which should give similar results for correlation times which do not badly violate Eq. (35), may bring to light many interesting effects. Implications of the density fluctuations discussed here on stellar collapse and supernova dynamics will be published elsewhere [17.

\section{ACKNOWLEDGMENTS}


We thank to G. Fuller, W. Haxton, and Y. Qian for very useful discussions. This research was supported in part by the U.S. National Science Foundation Grant No. PHY-9314131 and in part by the University of Wisconsin Research Committee with funds granted by the Wisconsin Alumni Research Foundation. F. N. L.'s research was supported in part by a grant from Mr. E. J. Loreti. We would also like to thank the Institute for Nuclear Theory at the University of Washington for its hospitality and the Department of Energy for partial support during the completion of this work.

\section{Appendix}

For $\hat{H}_{0}(t)$ given in Eq. (5), one selects the element of largest value during the time the system is in the presence of the random field. Let this largest value be $E_{\max }$. We consider the case where $\tau E_{\max } \ll 1$. We take $f_{i j}$ in Eqs. (10) and (11) to be given by,

$$
f_{i j}=\theta\left(\tau-\left|t_{i}-t_{j}\right|\right),
$$

where again the average of odd products vanish. We rewrite the average of Eq. (9) as,

$$
<\hat{\rho}_{I}(t)>=\hat{\rho}_{0}+<\hat{\rho}_{I}^{(2)}(t)>+<\hat{\rho}_{I}^{(4)}(t)>+<\hat{\rho}_{I}^{(6)}(t)>+\cdots
$$

where,

$$
\begin{aligned}
<\hat{\rho}_{I}^{(2 N)}(t)>= & (-1)^{N} \alpha^{2 N} \int_{0}^{t} d t_{2 N} \int_{0}^{t_{2 N}} d t_{2 N-1} \cdots \\
& \left.+\int_{0}^{t_{1}} d t_{1} F_{2 N}\left[\hat{M}\left(t_{2 N}\right),\left[\hat{M}\left(t_{2 N-1}\right), \cdots \hat{M}\left(t_{1}\right)\right] \cdots\right]\right]
\end{aligned}
$$

and,

$$
F_{2 N}=\sum_{n_{1} \cdots n_{2 N}}^{P(1,2, \cdots, 2 N)} f_{n_{1} n_{2}} f_{n_{3} n_{4}} f_{n_{5} n_{6}} \cdots f_{n_{2 N-1} n_{2 N}},
$$

where, $\mathrm{P}(1,2, \cdots, 2 \mathrm{~N})$ means all permutations. We explicitly show the second and third terms:

$$
\begin{array}{r}
<\hat{\rho}_{I}^{(2)}(t)>\sim-\alpha^{2} \int_{0}^{t} d t_{1}\left[\hat{M}\left(t_{1}\right), \int_{t_{1}-\tau}^{t_{1}}\left[\hat{M}\left(t_{1}\right)+\frac{d \hat{M}\left(t_{1}\right)}{d t}\left(t_{2}-t_{1}\right), \hat{\rho}_{0}\right]\right] \\
\sim-\alpha^{2} \tau \int_{0}^{t} d t_{1}\left[\hat{M}\left(t_{1}\right),\left[\hat{M}\left(T_{1}\right), \hat{\rho}_{0}\right]\right]+\alpha^{2} \frac{\tau^{2}}{2} \int_{0}^{t} d t_{1}\left[\hat{M}\left(t_{1}\right),\left[\frac{d \hat{M}\left(t_{1}\right)}{d t}, \hat{\rho}_{0}\right]\right],
\end{array}
$$

where $d \hat{M}\left(t_{1}\right) / d t=i \hat{U}_{0}^{\dagger}\left[\hat{H}_{0}, \hat{M}^{\prime}\right] \hat{U}_{0}$ and is therefore proportional to $E_{\max }$. In the third term one has a sum of three products of two f's, $\left(f_{12} f_{34}+f_{13} f_{24}+f_{14} f_{32}\right)$ only the first of which has the times in the order of the times appearing in the nested integrals. 
The first of these three terms gives,

$$
\begin{aligned}
<\hat{\rho}_{I}^{(4)}(t)>_{12,34} \sim & \alpha^{4} \tau^{2} \int_{0}^{t} d t_{1}\left[\hat{M}\left(t_{1}\right),\left\{\left[\hat{M}\left(t_{1}\right), \int_{0}^{t_{1}} d t_{2}\left[\hat{M}\left(t_{2}\right),\left[\hat{M}\left(t_{2}\right), \hat{\rho}_{0}\right]\right]\right]\right]\right. \\
- & \frac{\tau}{2}\left(\left[\frac{d \hat{M}\left(t_{1}\right)}{d t}, \int_{0}^{t_{1}} d t_{2}\left[\hat{M}\left(t_{2}\right),\left[\hat{M}\left(t_{2}\right), \hat{\rho}_{0}\right]\right]\right]\right] \\
+ & {\left.\left[\hat{M}\left(t_{1}\right), \int_{0}^{t_{1}} d t_{2}\left[\hat{M}\left(t_{2}\right),\left[\frac{d \hat{M}\left(t_{1}\right)}{d t}, \hat{\rho}_{0}\right]\right]\right]\right] } \\
& \left.\left.\left.+\left[\hat{M}\left(t_{1}\right),\left[\hat{M}\left(t_{1}\right),\left[\hat{M}\left(t_{1}\right), \hat{\rho}_{0}\right]\right]\right]\right]\right)\right\}+O\left(\tau^{4}\right) .
\end{aligned}
$$

The last term, however, is not proportional to $E_{\max }$. This will cause a problem since it could contribute to the next order term of the average. It turns out that it cancels the largest term coming from the remaining two f products. That these remaining $\mathrm{f}-$ products are of largest order $\tau^{3}$ can be seen by noting that when the argument of the theta function in an $\mathrm{f}$ connects two non-sequential times, the intermediate time(s) must also be within $\tau$ of the larger time in the theta function. For example, the remaining two contributions to $<\hat{\rho}_{I}^{(4)}(t)>$ are,

$$
\begin{aligned}
<\hat{\rho}_{I}^{(4)}(t)>_{13,24}+ & <\hat{\rho}_{I}^{(4)}(t)>_{14,23}= \\
& \alpha^{4} \int_{0}^{t} d t_{1}\left[\hat{M}\left(t_{1}\right),\left[\hat{M}\left(t_{1}\right),\left[\hat{M}\left(t_{1}\right),\left[\hat{M}\left(t_{1}\right), \hat{\rho}_{0}\right]\right]\right]\right] \\
\times & \int_{t_{1}-\tau}^{t_{1}} d t_{2} \int_{t_{1}-\tau}^{t_{2}} d t_{3}\left(\int_{t_{2}-\tau}^{t_{3}} d t_{4}+\int_{t_{1}-\tau}^{t_{3}} d t_{4}\right) \\
= & \alpha^{4} \frac{\tau^{3}}{2} \int_{0}^{t} d t_{1}\left[\hat{M}\left(t_{1}\right),\left[\hat{M}\left(t_{1}\right),\left[\hat{M}\left(t_{1}\right),\left[\hat{M}\left(t_{1}\right), \hat{\rho}_{0}\right]\right]\right]\right]
\end{aligned}
$$

This feature appears to continue throughout each term in the entire expression. Therefore, if $\tau E_{\text {max }} \ll 1$, and neglecting terms of order $\tau E_{\max }$ and smaller,

$$
\begin{aligned}
<\hat{\rho}_{I}^{(2 N)}(t)>= & (-1)^{N} \alpha^{2 N} \tau^{N} \int_{0}^{t} d t_{2 N}\left[\hat{M}\left(t_{2 N}\right),\left[\hat{M}\left(t_{2 N}\right)\right.\right. \\
& \int_{0}^{t_{2 N}} d t_{2 N-2}\left[\hat { M } \left(t_{2 N-2},\left[\hat{M}\left(t_{2 N-2}\right), \int_{0}^{t_{2 N-2}} \cdots\right.\right.\right. \\
& \left.\left.\left.\left.\int_{0}^{t_{4}} d t_{2}\left[\hat{M}\left(t_{2}\right),\left[\hat{M}\left(t_{2}\right), \hat{\rho}_{0}\right]\right] \cdots\right]\right]\right]\right]
\end{aligned}
$$

which leads to Eqs. (15) \& (16).

In the above derivation we have assumed $\alpha$ to be a constant. If $\alpha$ depends on time, the condition, $\tau E_{\max } \ll 1$, should be replaced by the condition,

$$
\tau\left[(d / d t \log \alpha(t))^{2}+E_{\text {max }}^{2}\right]^{\frac{1}{2}} \ll 1 .
$$




\section{References}

[1] L. Wolfenstein, Phys. Rev. D17, 2369 (1978); D20, 2634 (1979). S.P. Mikheyev and A. Yu. Smirnov, Nuovo Cim. 9C, 17 (1986); Sov. J. Nucl. Phys. 42, 913 (1986).

[2] W.C. Haxton, Phys. Rev. Lett. 57, 1271 (1986); S.J. Parke, ibid. 57, 1275 (1986); S.P. Rosen and J.M. Gelb, Phys. Rev. D 34, 969 (1986); A.B. Balantekin, S.H. Fricke, and P.J. Hatchell, Phys. Rev. D 38, 935 (1988); J.N. Bahcall and H.A. Bethe, Phys. Rev. Lett.65, 2233 (1990).

[3] C.-S. Lim and W.J. Marciano, Phys. Rev. D37, 1368 (1988). E.Kh. Akhmedov, Phys. Lett. B213, 64 (1988); E.Kh. Akhmedov and M. Yu. Khlopov, Mod. Phys. Lett. A3, 451 (1988).

[4] G. M. Fuller, R.W. Mayle, J.R. Wilson, and D.N. Schramm, Astrophys. J. 322, 795 (1987); Y.Z. Qian, G.M. Fuller, G.J. Mathews, R.W. Mayle, J.R. Wilson, S.E. Woosley, Phys. Rev. Lett. 71, 1965 (1993).

[5] R.F. Sawyer, Phys. Rev. D42, 3908 (1990).

[6] A. Schafer and S.E. Koonin, Phys. Lett. B 185, 417 (1987).

[7] W. C. Haxton and W-M. Zhang, Phys. Rev. D 43, 2484 (1991).

[8] P.I. Krastev and A. Yu. Smirnov, Phys. Lett. B 226, 341 (1989).

[9] A. Nicolaidis, Phys. Lett. B 262, 303 (1991).

[10] K. Enqvist and V. Semikoz, Phys. Lett. B 312, 310 (1993).

[11] N. G. Van Kampen, "Stochastic Processes in Physics and Chemistry," North Holland, (1990).

[12] J. N. Bahcall, W. F. Huebner, S. H. Lubow, P. D. Parker, and R. K. Ulrich, Rev. Mod. Phys. 54, 767 (1982), J. Bahcall and R. Ulrich, Rev. Mod. Phys. 60, 297 (1988); J.N. Bahcall and M.H. Pinsonneault, Rev. Mod. Phys. 64, 885 (1992).

[13] A. B. Balantekin, P. J. Hatchell, F. Loreti, Phys. Rev. D 41, 3583 (1990).

[14] X. Shi, D. N. Schramm, R. Rosner, and D. S. Dearborn, Commun. Nucl. Part. Phys. 21, 151 (1993).

[15] G. G. Raffelt, Phys. Rev. Lett. 64, 2856 (1990); Phys. Rep. 198, 1 (1990).

[16] H. Minakata and H. Nunokawa, Phys. Rev. Lett. 63, 121 (1989); A. B. Balantekin and F. Loreti, Phys. Rev. D 45, 1059 (1992).

[17] F.N. Loreti, Y. Qian, A.B. Balantekin, and G. Fuller, in preparation. 


\section{Figure Captions}

Figure $\mathbf{1}<\left|\psi_{1}(t)\right|^{2}>$ obtained by numerical solution of Eq. (21) (randomly fluctuating off-diagonal) with $B_{0}=1, k=0.05$ for (a) $A=0$, (b) $A=0.5$, (c) $A=2.0$ and (d) $A=10$. The initial condition is $\psi_{1}(0)=1, \psi_{2}(0)=0$.

Figure $\mathbf{2}<\left|\psi_{1}(t)\right|^{2}>$ obtained by numerical solution of Eq. (24) (randomly fluctuating diagonal) with $B_{0}=1, k=0.05$ for (a) $A=0$, (b) $A=0.5$, (c) $A=2.0$ and (d) $A=10$. The initial condition is $\psi_{1}(0)=1, \psi_{2}(0)=0$.

Figure $3 k \Delta r$ as a function of $\Delta m^{2} / E$ for a randomly fluctuating magnetic field of rms value $1 \times 10^{5} G$ confined to a region of the solar convective zone, as described in the text.

Figure $4<\left|\psi_{e}\right|^{2}>$ for infinite distance as a function of $\Delta m^{2} / E$. The lower(upper) curve around $\Delta m^{2} / E=1 \times 10^{-5}$ is the curve for a noiseless (noisy) electron density. The rms value of the randomly fluctuating noise is $2 \%$ of the local density. $\sin ^{2} 2 \theta$ is changed from (a) 0.8, (b) 0.1, (c) 0.01 and (d) 0.001. The correlation length is given by Eq. (35) for each value of $\Delta m^{2} / E$.

Figure 5 The same as Fig. (4) except that the rms value of the randomly fluctuating density is $4 \%$ of the local density. 


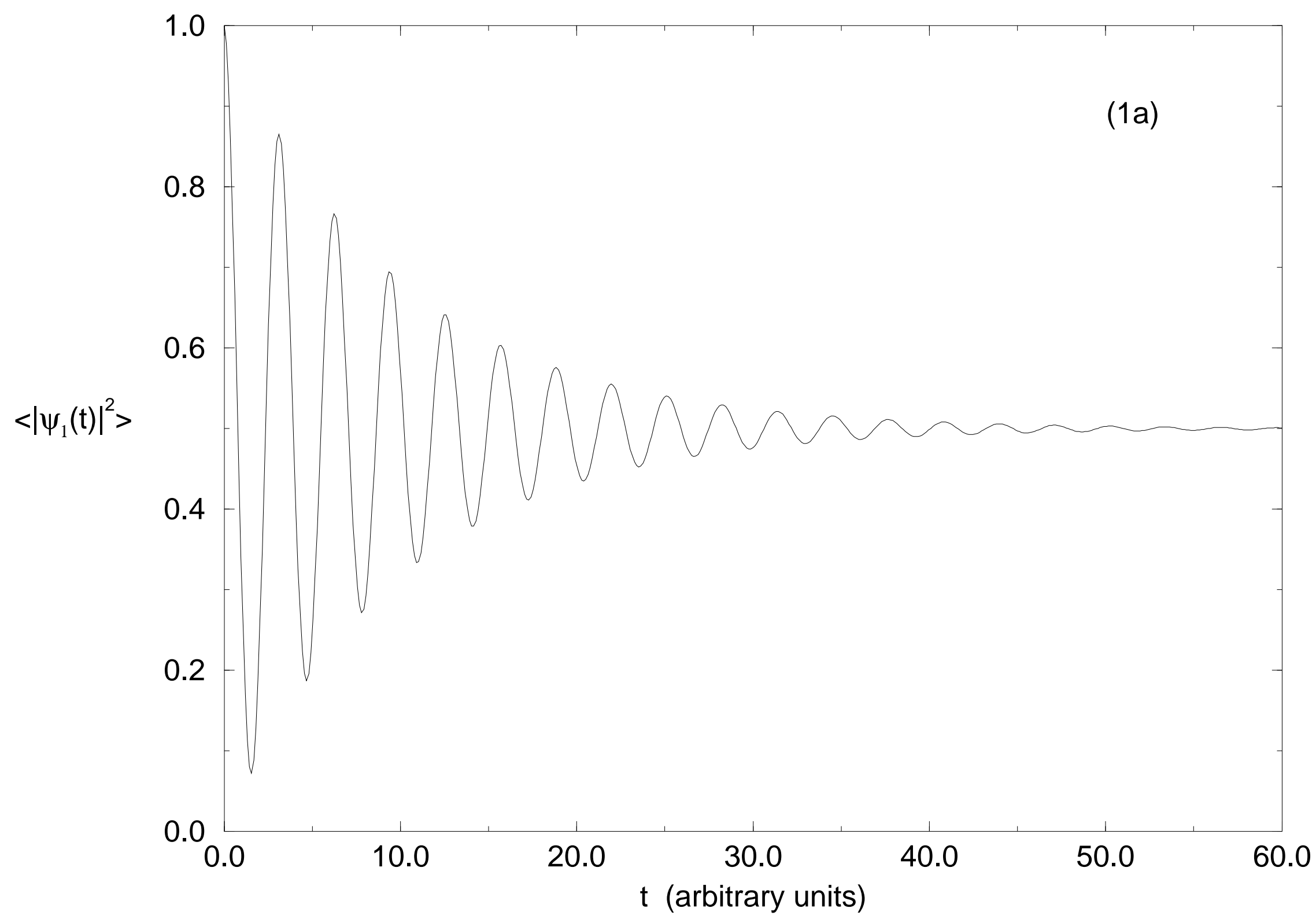




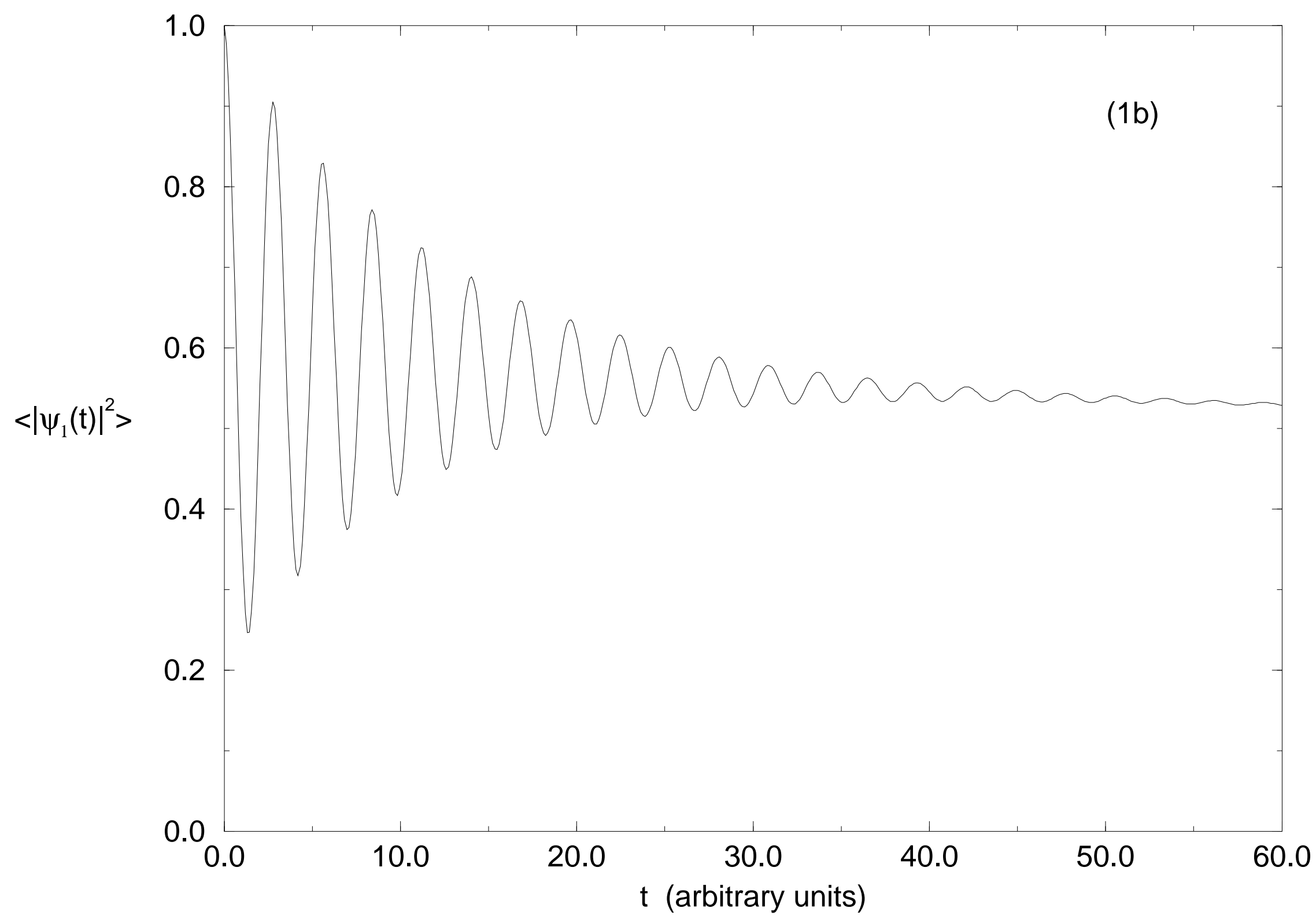




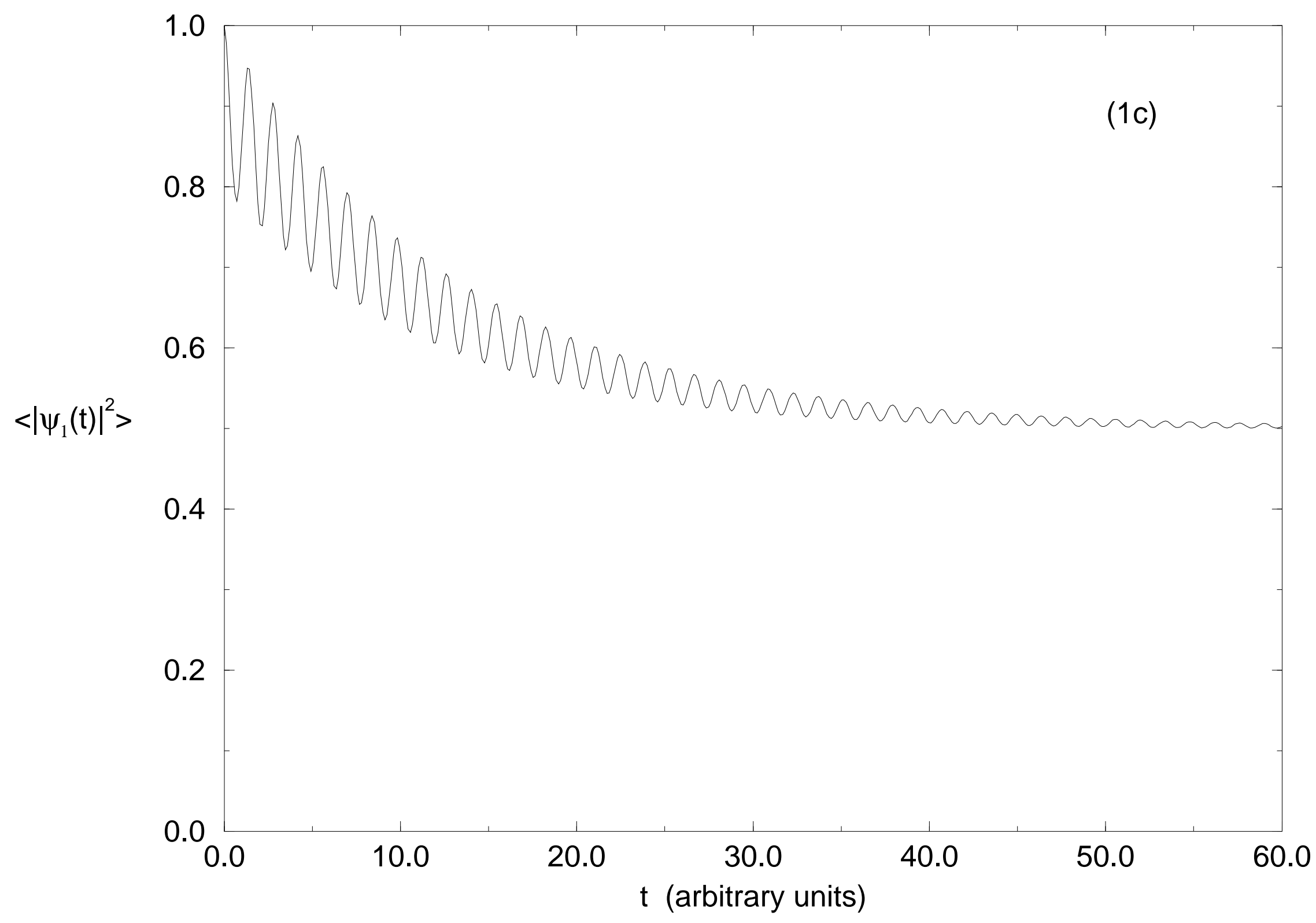




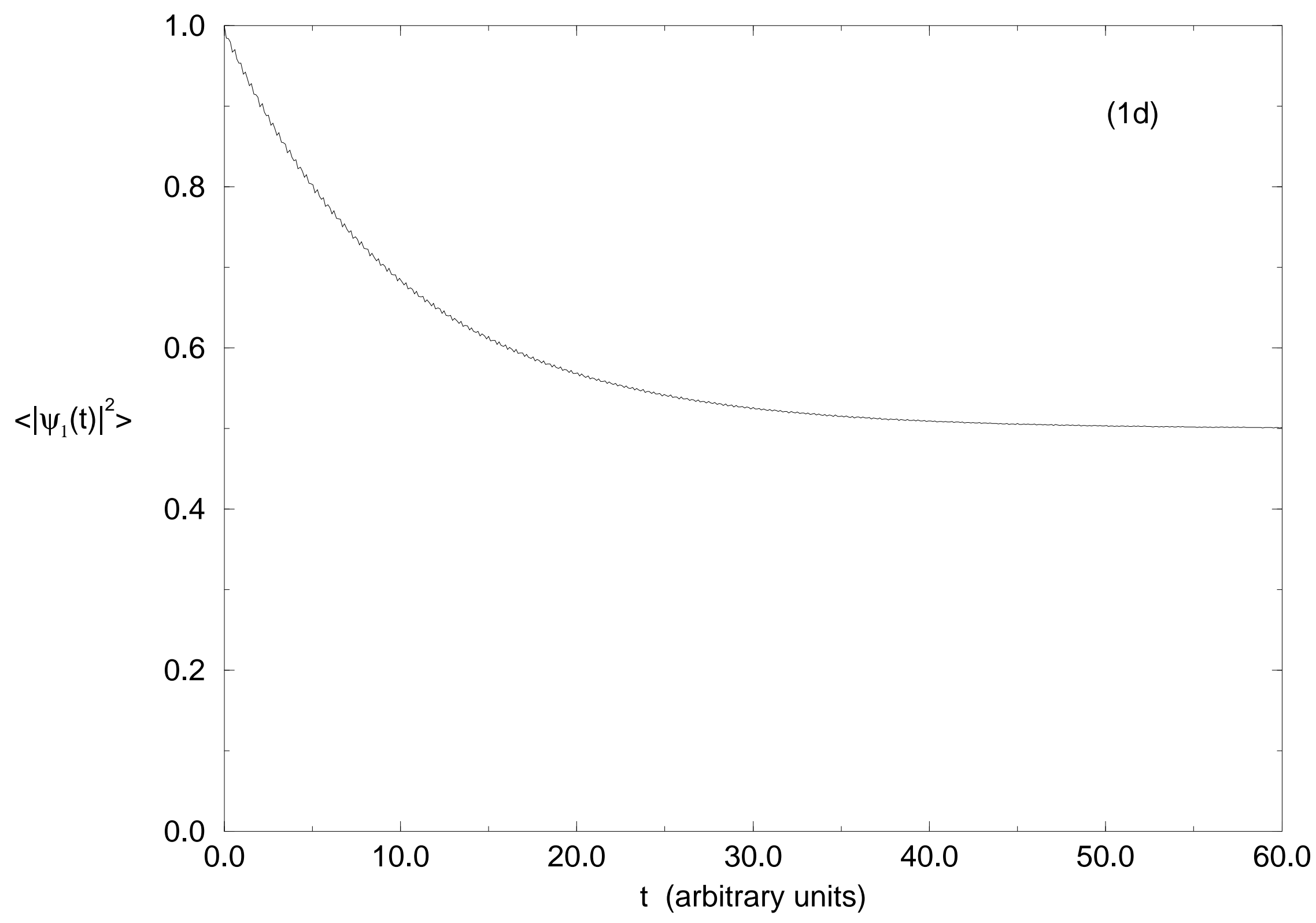




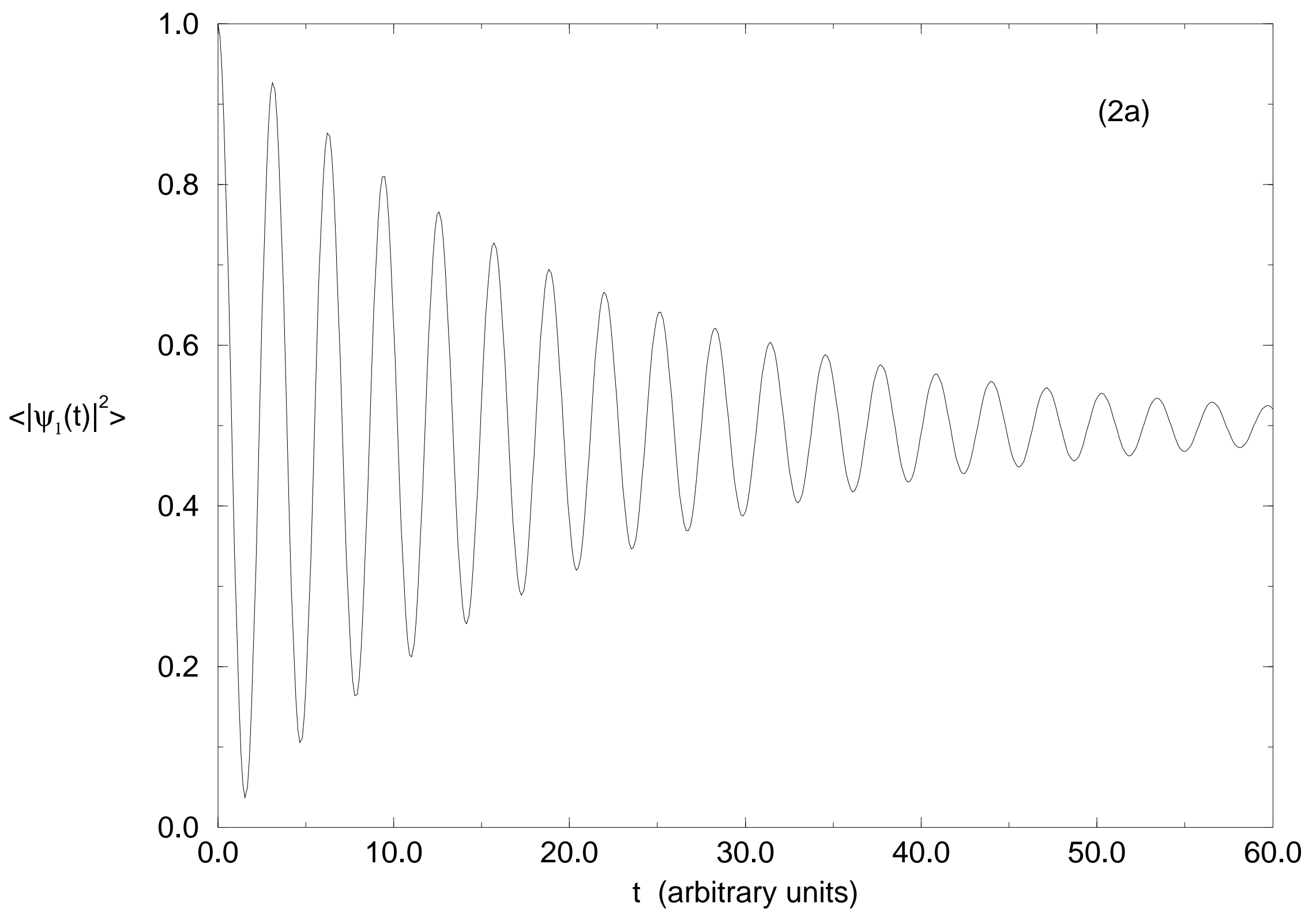




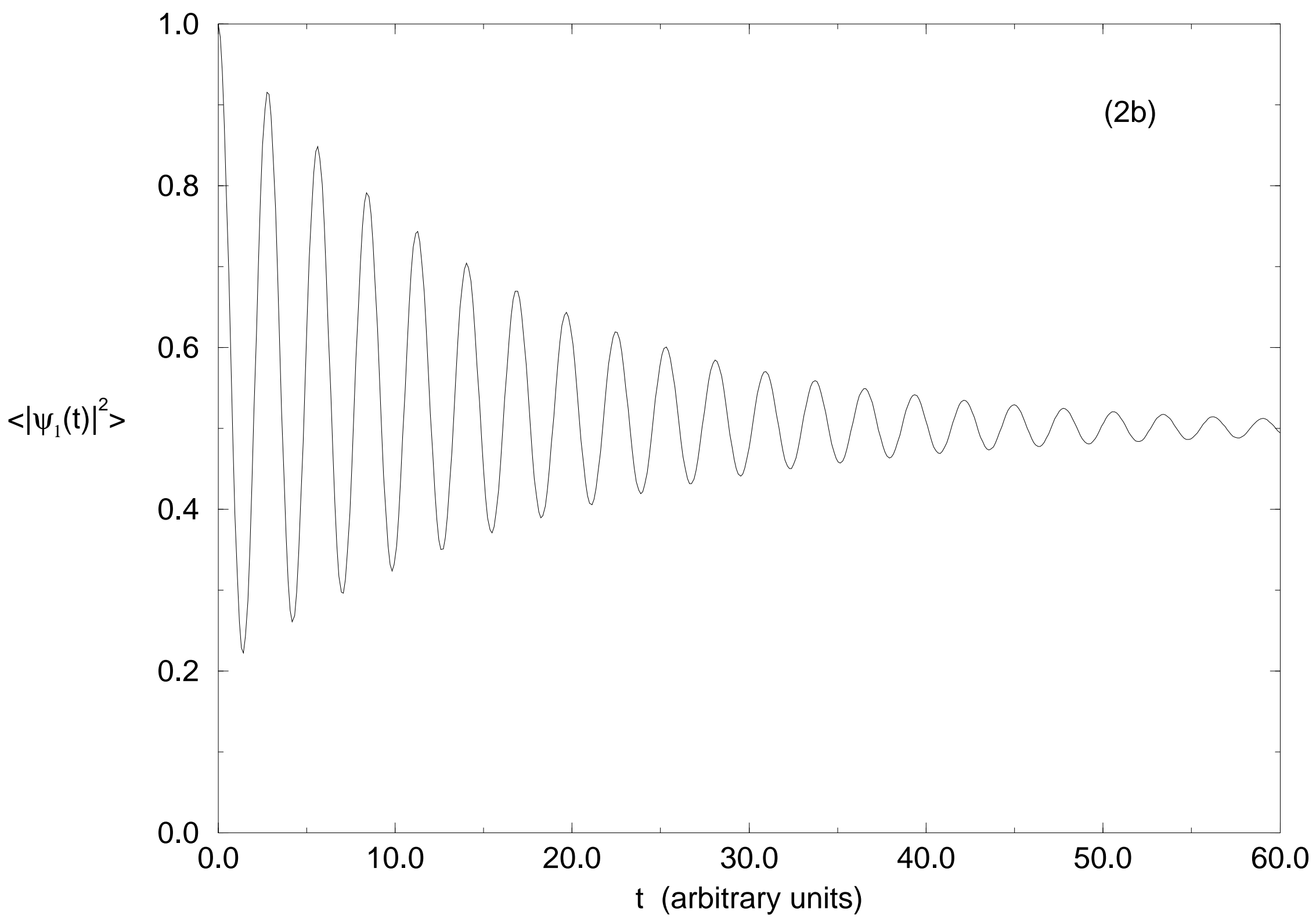




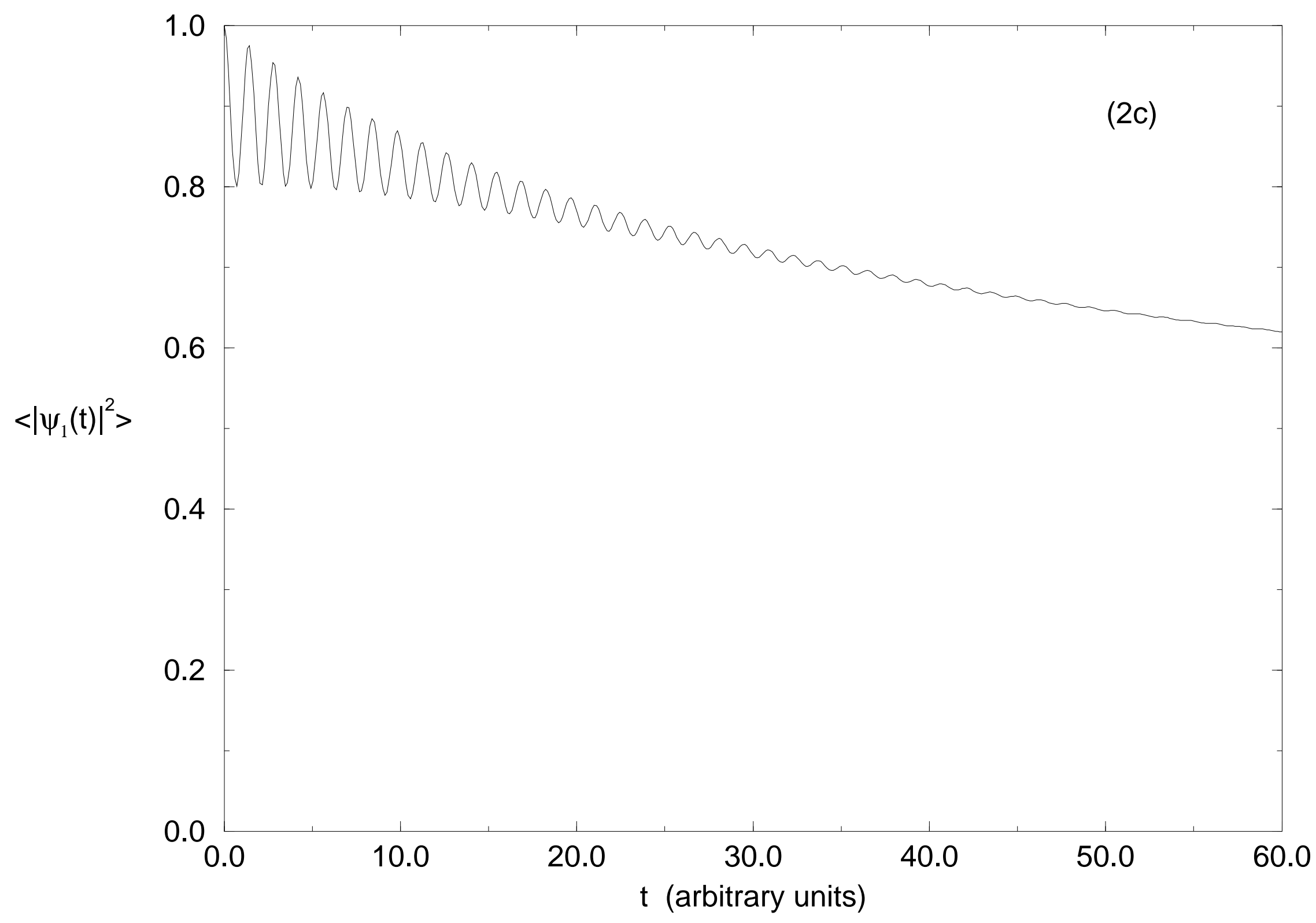




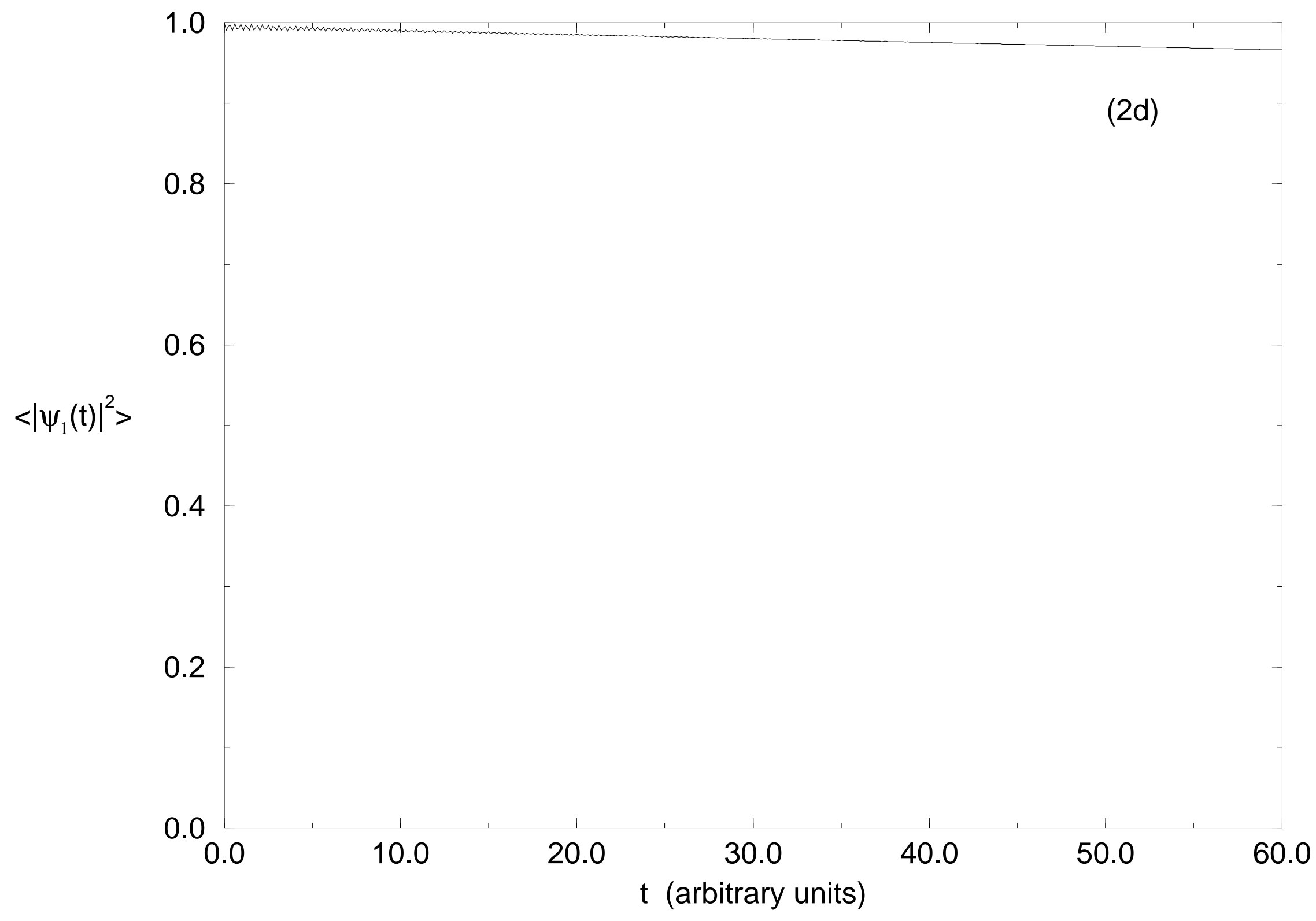




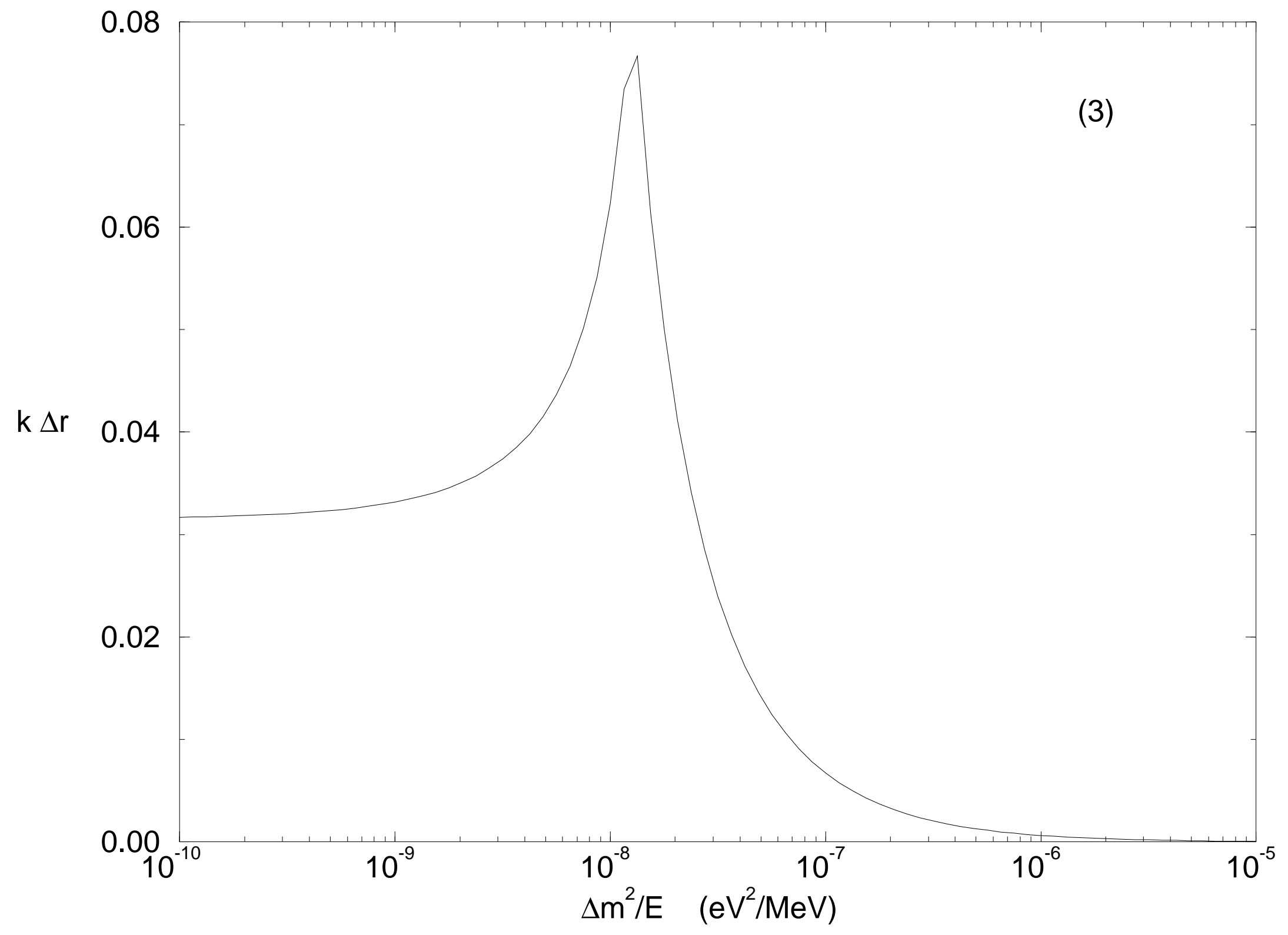




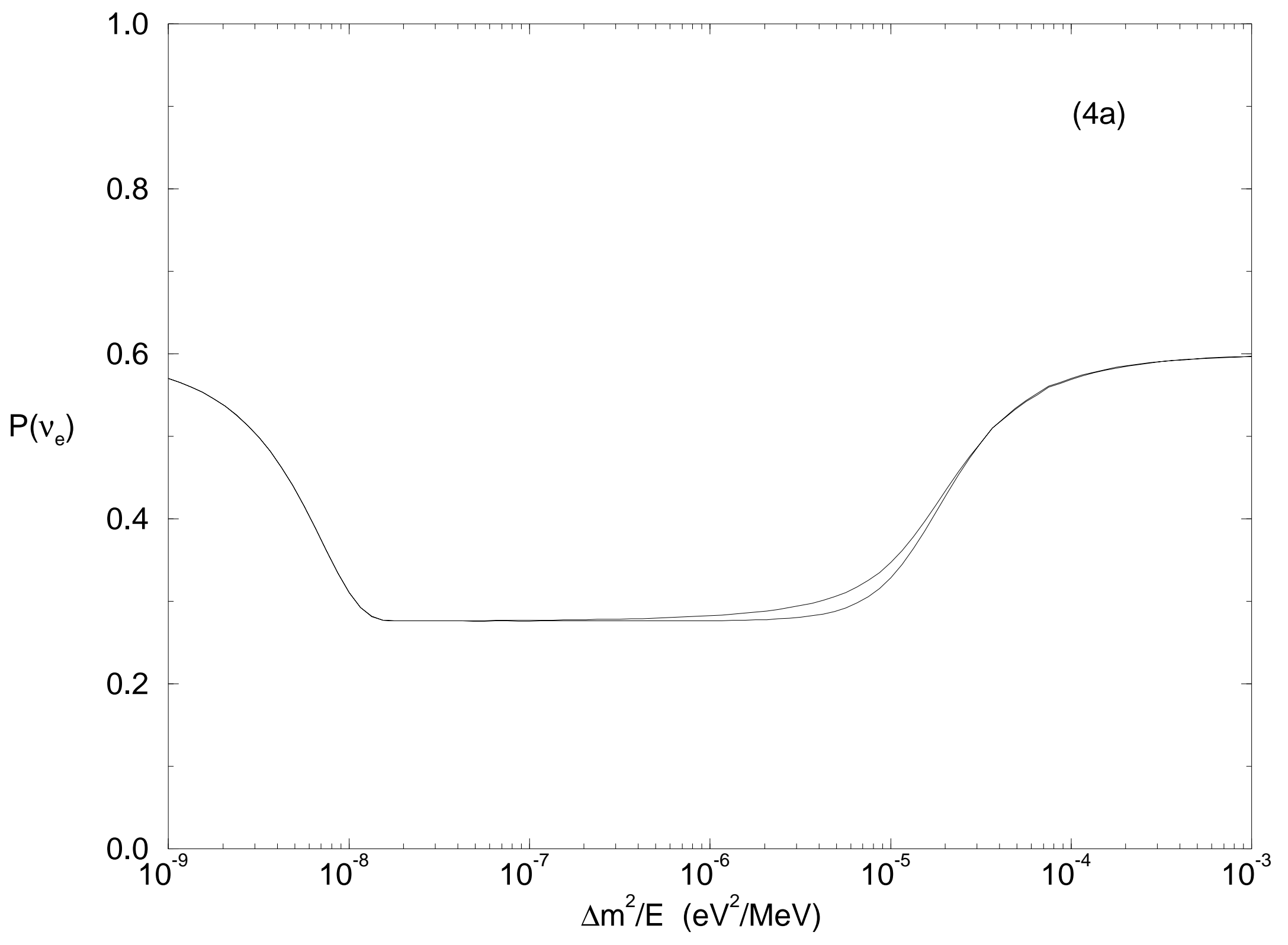




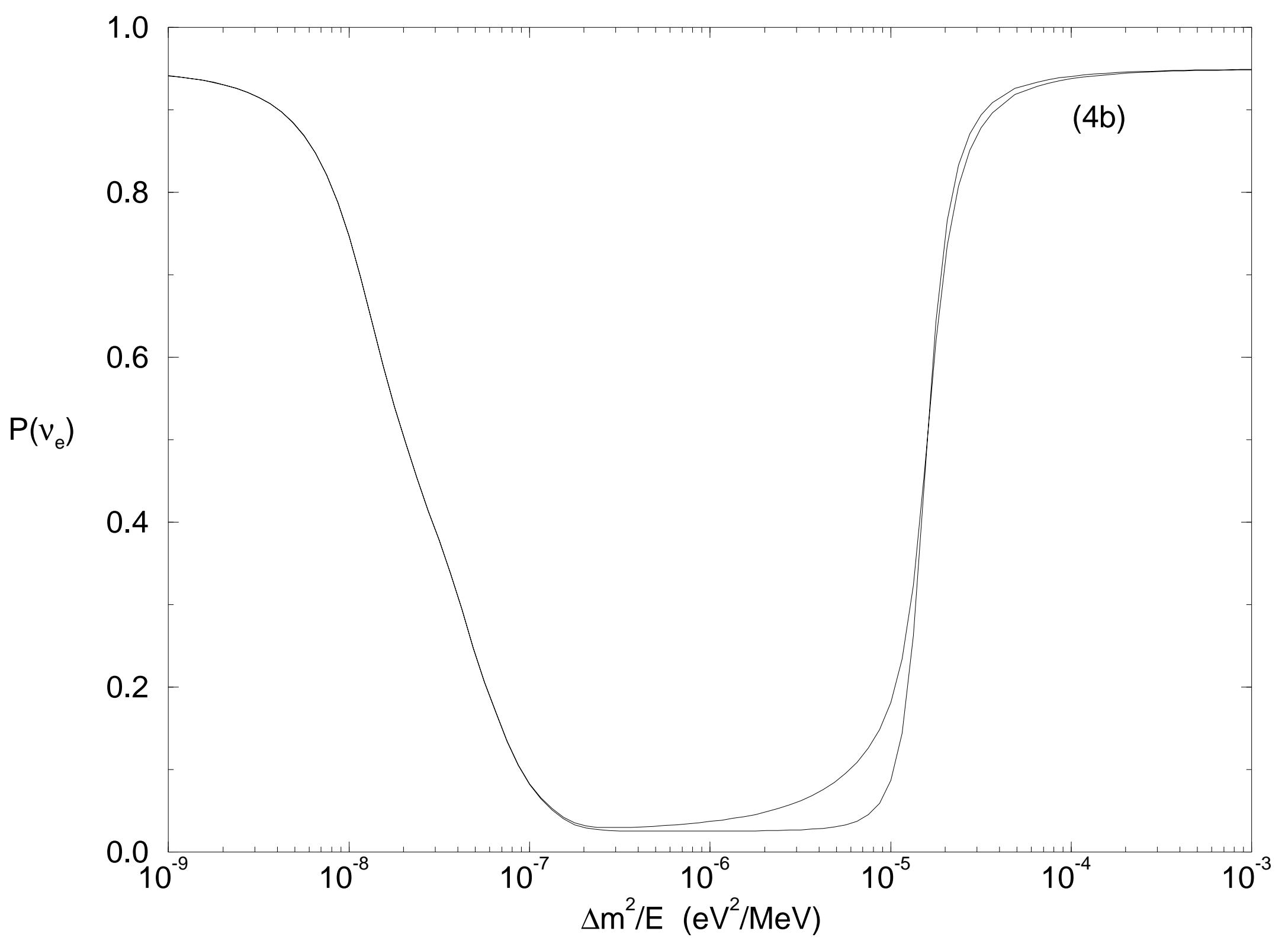




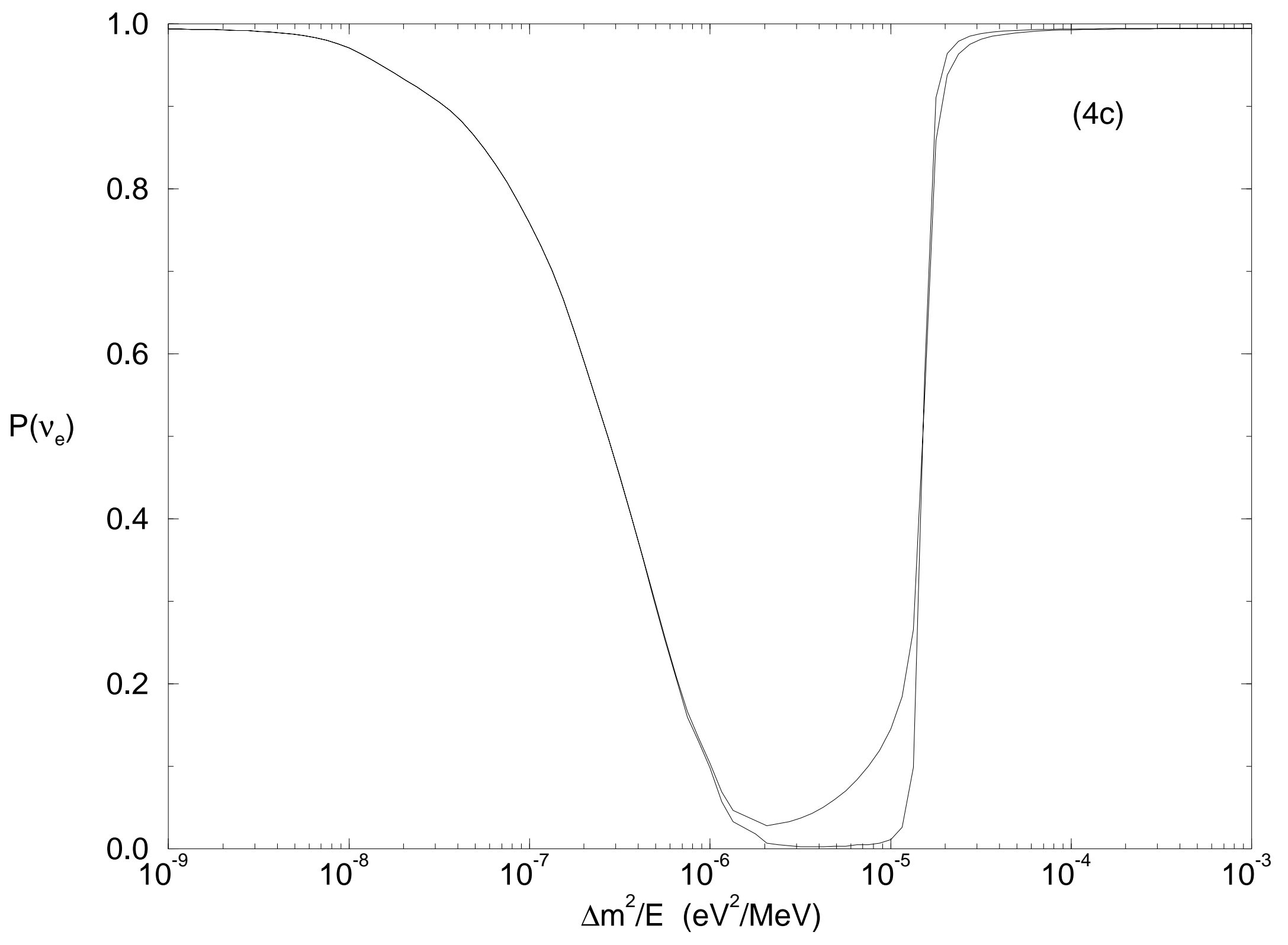




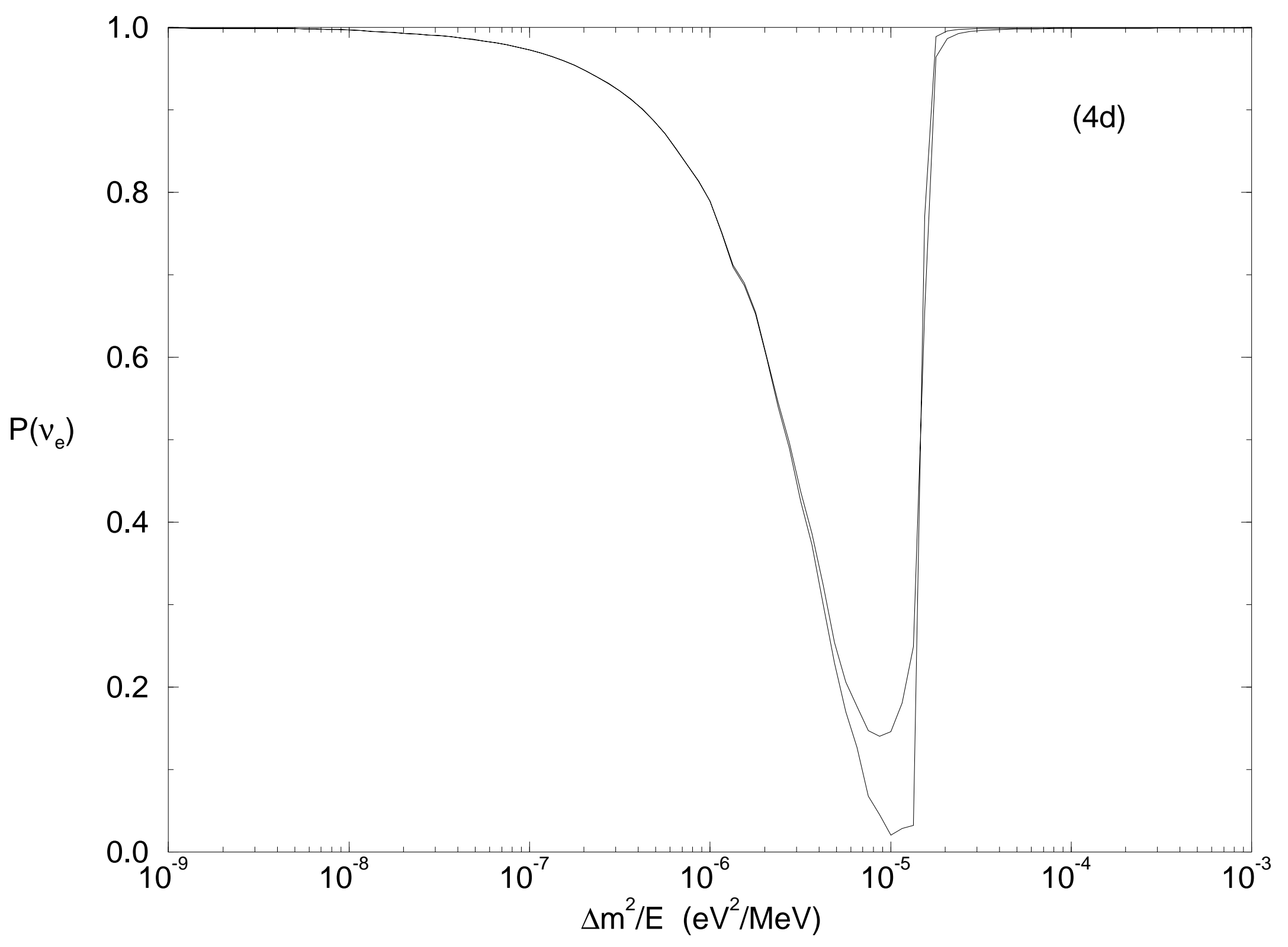




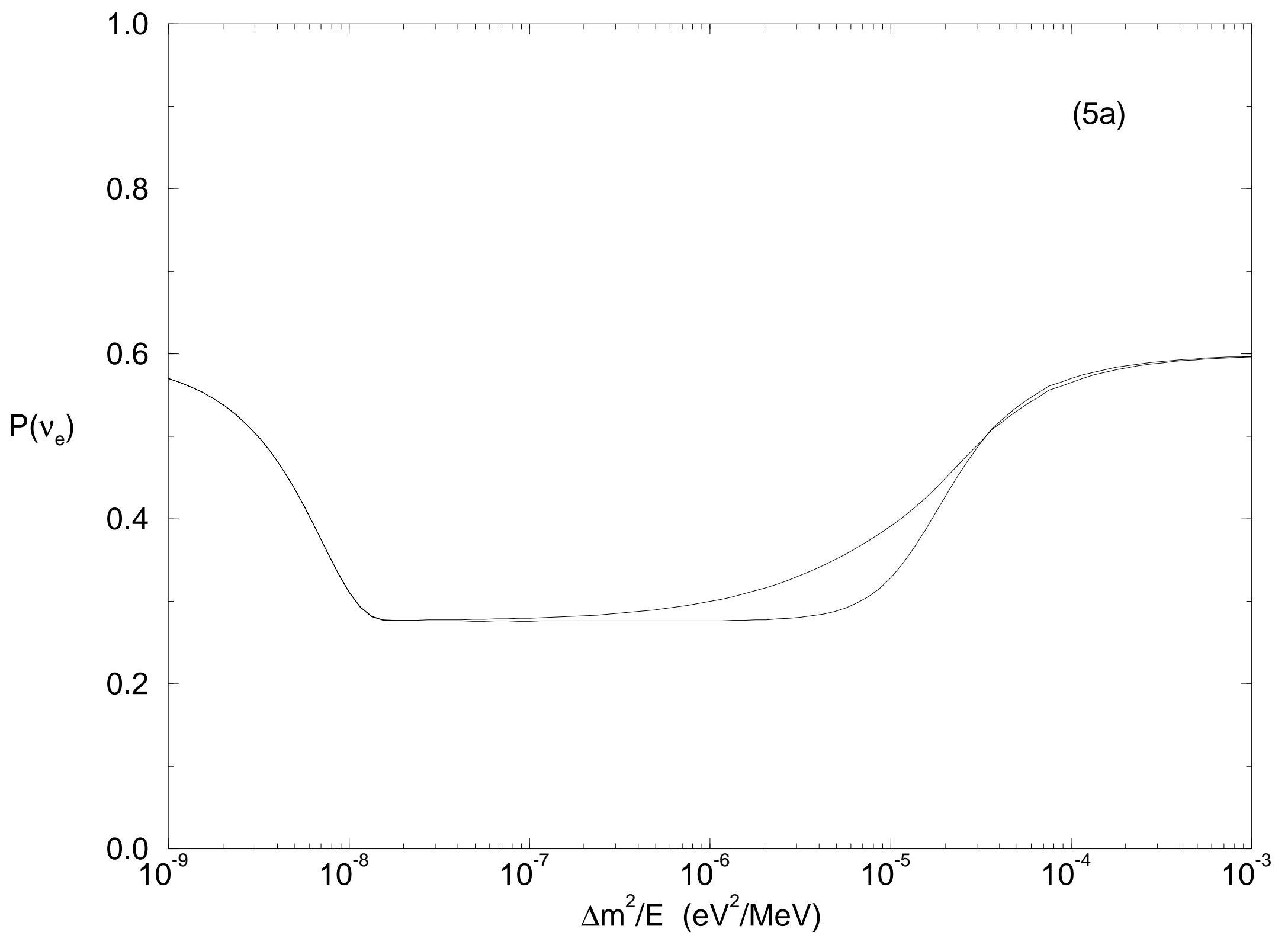




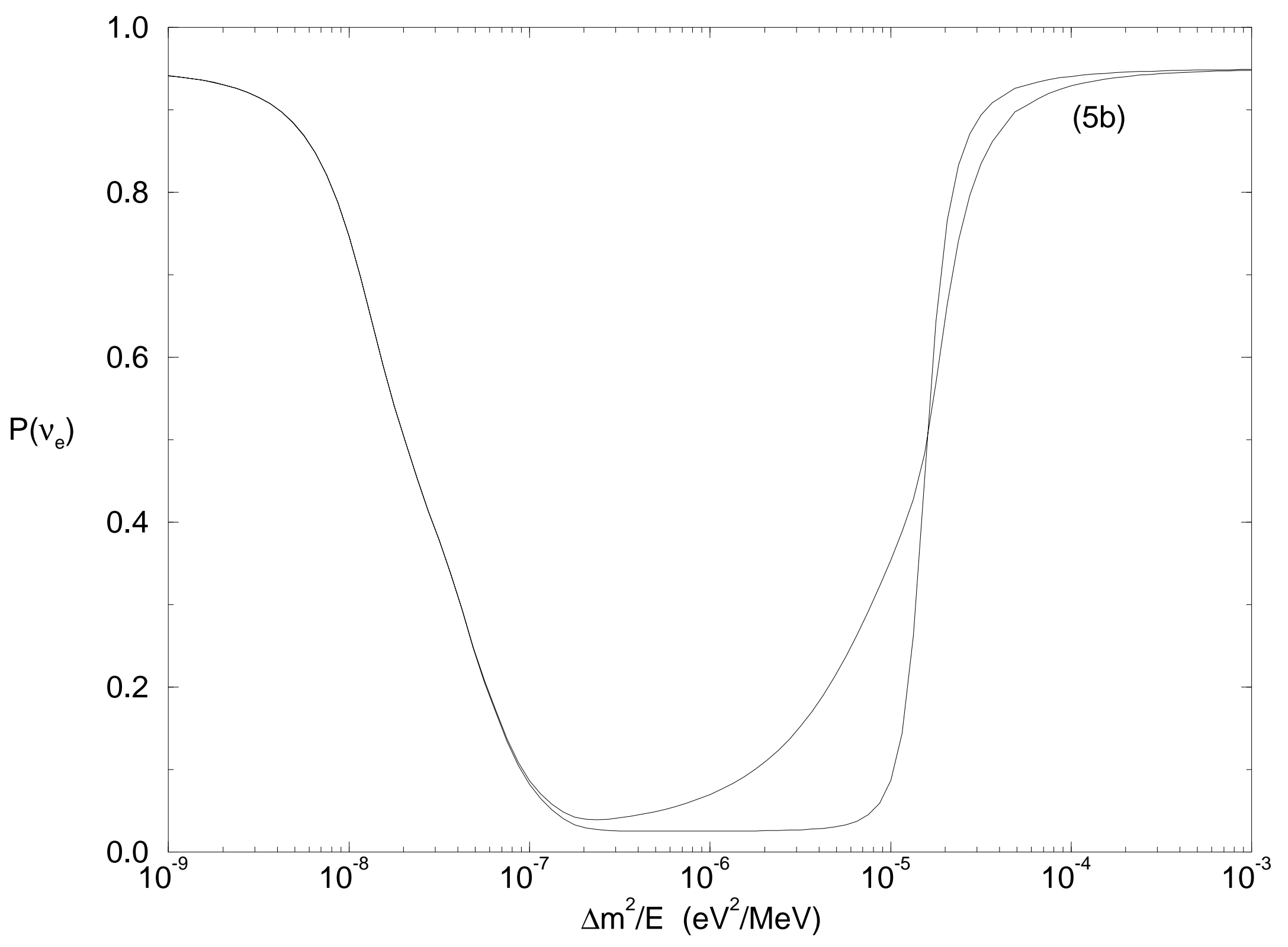




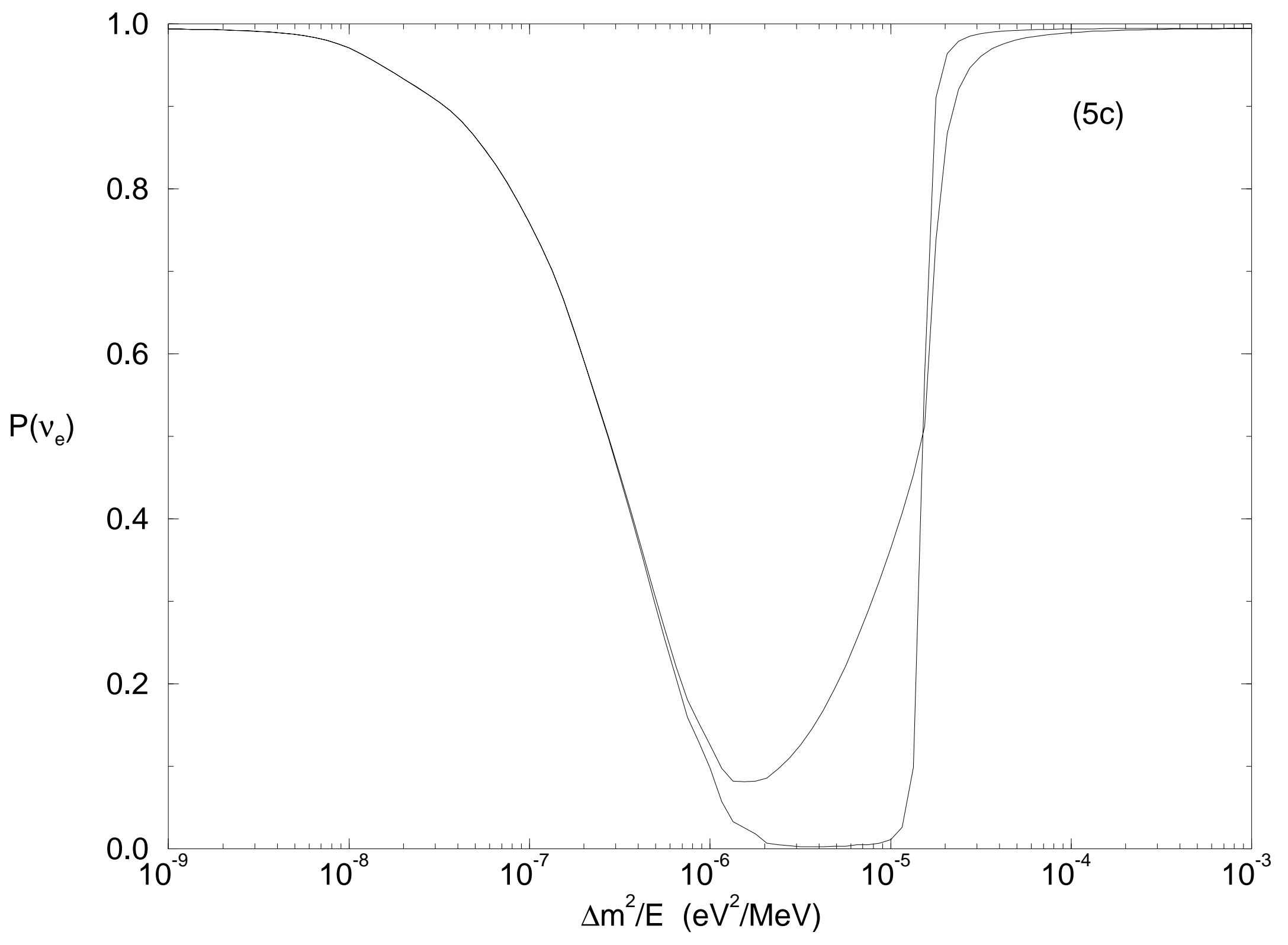




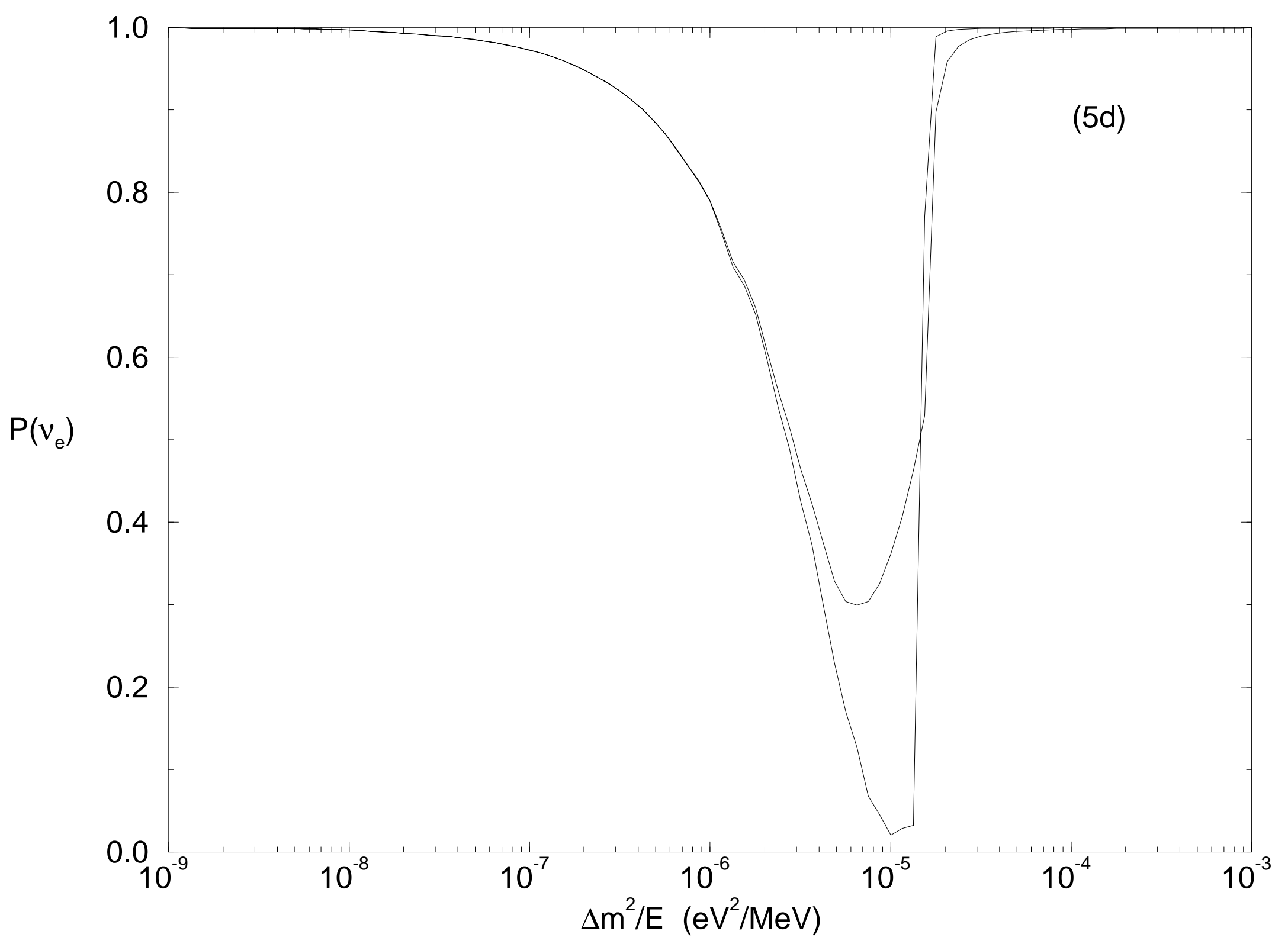

